\title{
Markers of cognitive reserve and dementia incidence in the English Longitudinal Study of Ageing
}

Pamela Almeida-Meza, Andrew Steptoe and Dorina Cadar

\section{Background}

In the current climate of an ageing population, it is imperative to identify preventive measures for dementia.

\section{Aims \\ We implemented a multifaceted index of cognitive reserve markers and investigated dementia incidence over 15 years of follow-up in a representative sample of the English population.}

\section{Method}

Data were 12280 participants aged $\geq 50$ years from the English Longitudinal Study of Ageing, free from dementia at their baseline assessments during wave 1 (2002-2003), 3

(2006-2007) or 4 (2008-2009), and followed up until wave 8 (2016-2017). The Cognitive Reserve Index was constructed as a composite measure of education, occupation and leisure activities, using a standardised questionnaire. Cox proportional hazards regression models were used to estimate the hazard ratios of dementia in relation to cognitive reserve levels (low, medium and high) and its components (education, occupation and leisure activities).

\section{Results}

During the follow-up period, 602 participants aged 56-99 years developed dementia. Higher levels of cognitive reserve (hazard ratio $0.65,95 \% \mathrm{Cl} 0.48-0.89, P=0.008$ ) were associated with a lower risk of dementia. An individual analysis of its components showed that higher levels of education (hazard ratio $0.56,95 \% \mathrm{Cl}$
$0.36-0.88, P=0.012$ ), occupation (hazard ratio $0.72,95 \% \mathrm{Cl} 0.56-$ $0.91, P=0.008$ ) and leisure activities (hazard ratio $0.74,95 \% \mathrm{Cl}$ $0.56-0.99, P=0.047$ ) were predictive of a reduced dementia risk, with the first two components particularly protective in younger participants ( $<85$ years).

\section{Conclusions}

This study showed a reduced risk of dementia for individuals with a higher level of cognitive reserve, represented by higher education, complex occupations and multifaceted level of leisure activities.

\section{Declaration of interest}

None.

\section{Keywords}

Dementia; clinical neurology; cognitive reserve; leisure activities; ageing.

\section{Copyright and usage}

(C) The Royal College of Psychiatrists 2020. This is an Open Access article, distributed under the terms of the Creative Commons Attribution-NonCommercial-NoDerivatives licence (http://creativecommons.org/licenses/by-nc-nd/4.0/), which permits noncommercial re-use, distribution, and reproduction in any medium, provided the original work is unaltered and is properly cited. The written permission of Cambridge University Press must be obtained for commercial re-use or in order to create a derivative work.
Dementia represents one of the major contributors to disability and dependency among the elderly population, imposing significant challenges over society's welfare and healthcare systems. ${ }^{1}$ Consequently, it is crucial to identify preventive measures to maintain cognitive health and reduce dementia risk. The concept of $\operatorname{cog}$ nitive reserve has been proposed to account for individual differences in the susceptibility to cognitive impairment related to neuropathological damage. Cognitive reserve appears to provide the ability to mask neurocognitive deficits, providing a protective effect against dementia risk. ${ }^{2}$ This theory suggests that individuals with higher cognitive reserve show less cognitive and functional impairment because their cognitive networks are more efficient, capable and flexible. ${ }^{3,4}$ Hence, cognitive reserve is often considered as a potential moderator between brain damage and exhibited cognitive impairment. Epidemiological evidence has shown that cognitive reserve capacity may be modified through lifetime activities such as education, occupation and leisure activities. ${ }^{3}$ However, there are important limitations to the investigation of cognitive reserve in population studies. First, there is a lack of consensus on the definition of cognitive reserve and how to assess its determinants. ${ }^{5}$ Second, most published studies in this area are either cross-sectional or based on small samples. Third, there is an imperative need to understand the role of modifiable cognitive reserve markers to promote optimal cognitive health and support the prevention of dementia.
The present study sought to implement a detailed measurement of cognitive reserve markers in a longitudinal study of ageing by applying a multifaceted index in a population-based cohort of older adults in England. The overall aim of this study was to investigate the association between the Cognitive Reserve Index, derived by Nucci et $\mathrm{al}^{6}{ }^{6}$ and its components in relation to subsequent dementia incidence. Based on the previous literature, it was hypothesised that (a) an increased Cognitive Reserve Index score will have a protective effect against dementia risk, and (b) an increased level of each cognitive reserve marker will be associated with reduced dementia risk.

\section{Method}

\section{Study population}

The data were extracted from the English Longitudinal Study of Ageing (ELSA), which is a longitudinal panel study of a representative sample of initially non-institutionalised people living in England, aged $\geq 50$ years. ELSA was designed to collect data on a range of multidisciplinary topics relevant to the ageing process. Data collection was carried out every two years, with refreshment samples joining the study at different stages. The baseline for the present analysis was either wave 1 (2002-2003) for those who started the study at wave 1 , or wave 3 


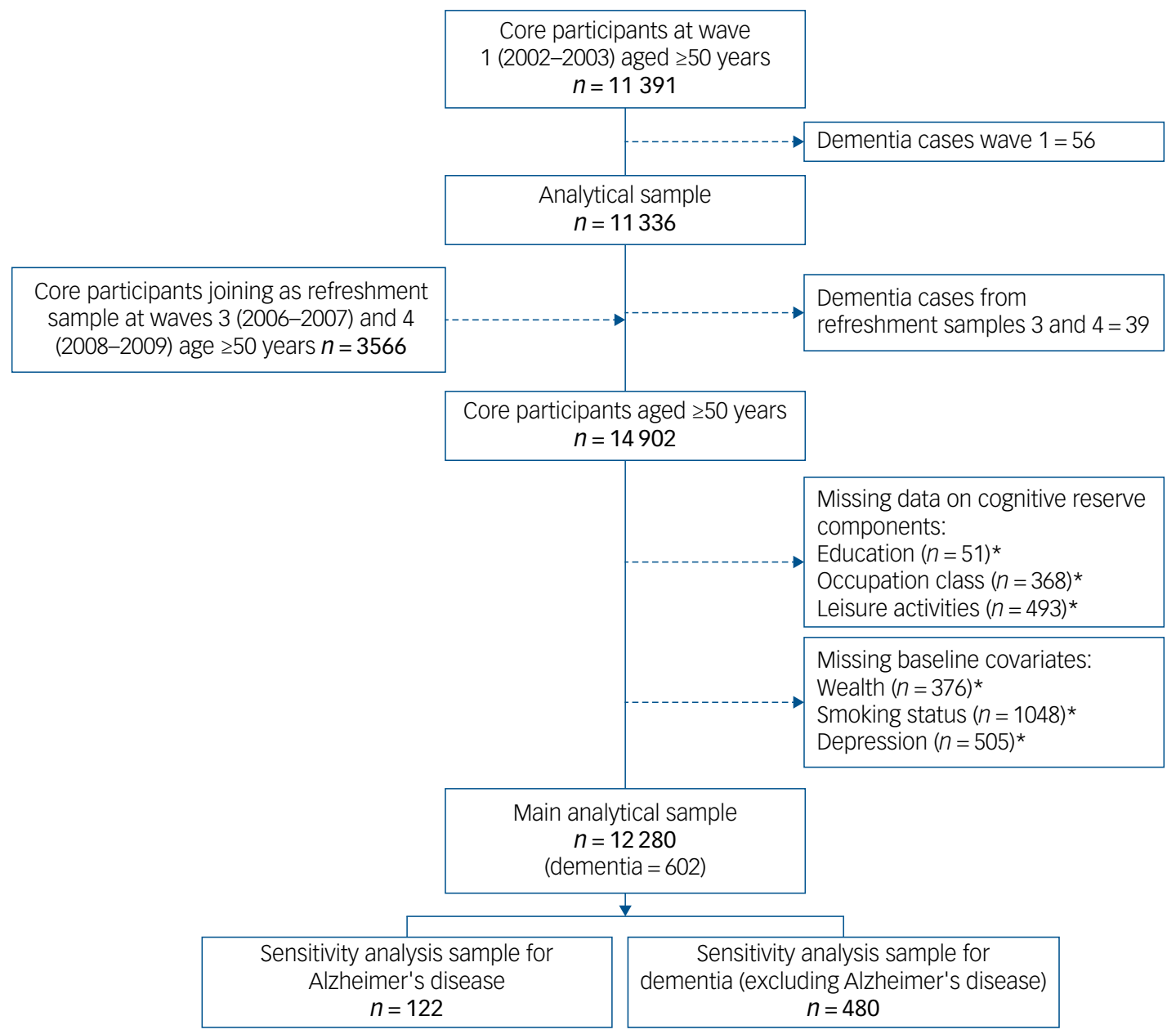

Fig. 1 Flow chart of participants from the English Longitudinal Study of Ageing included in analyses.

*Numbers of excluded participants are non-mutually exclusive.

(2006-2007) or 4 (2008-2009) for those who joined the study as refreshment samples. The latest wave available at the time of this analysis was wave 8 (2016-2017), ensuring a follow-up period of up to 15 years. Participants with dementia at their baseline assessments were excluded. See Fig. 1 for analytical sample flow chart.

\section{Ethics}

This study was approved by the National Research Ethics Committee (reference number MREC/01/2/91). All participants provided informed consent to take part in this study.

\section{Dementia}

In ELSA, dementia diagnosis was ascertained at each wave through a combination of self-report physician diagnosis of dementia or Alzheimer's disease, and/or a higher score on the Informant Questionnaire on Cognitive Decline in the Elderly (IQCODE). ${ }^{7}$ This questionnaire was completed by a family member or long-term caregiver. A score above the threshold of 3.38 in the IQCODE is considered indicative of pathological cognitive decline. This threshold has both high specificity $(0.84)$ and sensitivity $(0.82){ }^{8}$

\section{Cognitive Reserve Index questionnaire}

Cognitive reserve was measured with the Cognitive Reserve Index questionnaire (CRIq) devised by Nucci et $\mathrm{al}^{6}{ }^{6}$ and designed to quantify various markers of cognitive reserve, providing a standardised reflective measure of the cognitive reserve acquired during a person's lifetime. The Cognitive Reserve Index is a composite measure of educational attainment, occupational class and leisure activities. These three markers provide the CRIq with good reliability ( $\alpha=0.62,95 \%$ CI 0.56-0.97). ${ }^{6}$ The data for each component were extracted from various self-completion questionnaires administered to each core member during their baseline assessment (Supplementary Table 1 available at https://doi.org/10.1192/bjp.2020.54).

\section{Education}

Education attainment was categorised into four groups, each representing an approximate amount of years spent in formal education: having a university degree or higher, 15 years; having completed A levels or the equivalent (vocational specialisations, work-related training and advanced level qualifications obtained after secondary education), 12 years; having completed education to School Certificate level (taken at age 15-16 years), 8 years; and lacking formal qualifications, 4 years.

\section{Occupation}

Occupational class was categorised according to the National Statistic Socio-economic Classification into five categories: 
low-skilled manual work, skilled manual work, skilled non-manual work, professional occupation and highly responsible or intellectual occupation. ${ }^{9}$ The original CRIq uses the number of years spent on work occupation and multiplies it by the level of the category. ${ }^{6}$ However, in ELSA, the number of years was not specifically assessed, and so we conducted our main analysis without this element.

\section{Leisure activities}

Leisure activities were measured by 17 questions as per Nucci et al, ${ }^{6}$ which have shown good reliability $(\alpha=0.73,95 \%$ CI $0.70-0.76){ }^{6}$ This range of leisure activities was reflected by four levels of frequency: weekly (e.g. reading the newspaper), monthly (e.g. social activities), annual (e.g. journeys/trips) and fixed activities (e.g. pet care). In ELSA, pet tenancy information was only available at wave 5 (2010-2011), from where this information was extracted. Furthermore, participants were not asked whether they read books, so this question was not included. Finally, to calculate the raw score of this component, the original CRIq multiplies each of the leisure activities by the number of years participants engaged in the activities; however, in ELSA, we used a calculation of points based on each of the 16 activities performed without an estimation of years.

For the computation of occupation and leisure activities, linear regressions were carried out with baseline age as the predictor variable. The residuals of the two linear models were divided by the s.d. of the sample, standardised and transposed to a scale with a mean of 100 and an s.d. of $15 .^{6}$

\section{Overall Cognitive Reserve Index}

To derive an overall index of various markers of cognitive reserve acquired throughout an individual's lifespan, we averaged the scores of the three individual markers and standardised and transposed them to a scale with a mean of 100 and an s.d. of 15, as per Nucci et al. ${ }^{6}$ We only included participants with complete data for age, education, occupation and at least two leisure activities items. In the present study, the overall Cognitive Reserve Index ranged between 60.6 and 150 and was classified according to the thresholds used by Nucci et al, ${ }^{6}$ (e.g. low, $\leq 70$; medium-low, 7184; medium, 85-114; medium-high, 115-129 and high, $\geq 130$ ). However, given the limited number of participants captured into the highest and lowest categories in our sample, we re-grouped the top two highest and lowest categories, resulting into following three groups: low $(\leq 84)$, medium $(85-114)$ and high $(\geq 115)$ (Supplementary Table 2).

\section{Covariates}

Being female or unmarried has been identified as having an increased risk of developing dementia; ${ }^{10,11}$ hence, gender and marriage were included as covariates. Wealth quintiles were included because previous ELSA findings showed an association between lower wealth and dementia. ${ }^{12}$ The measure of wealth reflects the accumulation of assets over the life course; the variable includes financial wealth, the value of properties, business assets and physical wealth minus any debt. The median wealth was approximately $£ 120$ for participants in the lowest quintile, and $£ 180000$ for those in the highest quintile. The baseline median wealth for the overall sample included in these analyses was $£ 15100 .{ }^{12}$ Furthermore, there is an established association between depression and dementia; ${ }^{13}$ therefore, we included depressive symptoms ascertained with a score of 4 or higher on the eight-item Center for Epidemiologic Studies Depression Scale. ${ }^{14}$ Finally, because health behaviours and poor physical health are associated with an increased risk of dementia, ${ }^{13,15}$ smoking status and physician diagnosis of coronary heart disease (CHD; including myocardial infarction and angina), stroke, hypertension and diabetes were included as physical health covariates.

\section{Statistical analysis}

Descriptive statistics were examined using Pearson's $\chi^{2}$-tests to determine if there were significant differences in baseline characteristics between participants who developed dementia and those who remained dementia-free.

Cox proportional hazards regression models were used to estimate the hazard ratios and confidence intervals of dementia incidence in relation to Cognitive Reserve Index and its components. Age was used as the underlying time variable for the survival analyses considered here within the study period, ranging from wave 1 until the time of dementia onset or last wave of follow-up (wave 8) if not diagnosed with dementia. For individuals who died or dropped out, right censoring was applied considering the survival age at their last wave of data available. Attrition rates are presented in Supplementary Table 3.

Nine models were fit to the data. Model 1 examined the relationship between Cognitive Reserve Index and dementia incidence, controlling for gender and marital status. Models 2-8 were based on model 1 , with additional adjustment for wealth, smoking status, depressive symptoms, CHD, diabetes, stroke and hypertension, respectively; model 9 adjusted for all covariates. The inflation factor was $<2.01$, suggesting no significant multicollinearity. Proportional hazards assumptions for the Cognitive Reserve Index were checked using Schoenfeld residuals. The Cognitive Reserve Index $(P=$ $0.179)$ and occupation subcomponent $(P=0.475)$ met the proportional hazards assumption. However, these assumptions were not fulfilled for education and leisure activities. Separate models were carried out before and after 80 years of age for education, and before and after 85 years of age for leisure activities.

Additionally, to assess the extent to which baseline risk factors explained the association of cognitive reserve with dementia incidence, the percentage of excess risk mediated (PERM) was calculated for each one of the risk factors included: wealth, smoking status, depressive symptoms, CHD, diabetes, stroke, and hypertension. The PERM ${ }^{16}$ was estimated as:

\section{PERM $=$}

hazard ratio (model 1) - hazard ratio(model $1+$ risk factor) hazard ratio(model 1$)-1 \quad \times 100$.

The baseline cross-sectional weights derived in ELSA were used in all analyses to ensure the sample is representative of the English population. We used STATA SE, version 14 (StataCorp) for Windows to carry out all analyses. The statistical significance was considered at standard levels at or below 0.05 .

\section{Sensitivity analyses}

Several sensitivity analyses were conducted. The first two investigated Cognitive Reserve Index in relation to dementia types (Alzheimer's disease and other types of dementia). The third and fourth explored the categorisation of cognitive reserve with different thresholds by dividing the cognitive reserve into tertiles and quintiles. The fifth analysis used an overall Cognitive Reserve Index for which the occupation was calculated by multiplying the score corresponding to each level of working activity by the number of years estimated from 40 years of age until 65 years of age (considered as the retirement age), or until the participant's baseline age if they were younger than 65 years at the beginning of the study. 
Finally, the sixth sensitivity analysis was conducted to further control for apolipoprotein E e4 locus (APOEe4) genotype and baseline alcohol consumption in a subset analytical sample, owing to a low number of observations for each (APOEe4 $n=6799$ and alcohol consumption $n=7697$ ). We also tested the interaction between APOEe4 and cognitive reserve.

\section{Results}

\section{Descriptive statistics}

The analytical sample comprised 12280 individuals free form dementia at baseline, accounting for 114234 person-years. The sample consisted of 5626 men and 6654 women, with a mean age of $63.66 \pm 9.8$ (s.d.) ranging from 50 to 100 years at baseline. At the time of the event or last wave of follow-up, the mean (s.d.) age for all participants was $72.96 \pm 9.7$ years, ranging from 52 to 108 years. From the overall sample, 602 participants were diagnosed with dementia, accounting for a $5 \%$ cumulative incidence during the 15 -year follow-up period. The group diagnosed with dementia included 251 men (2\%) and 351 women (2.8\%), with the median age of $80.8 \pm 8.2$ years at the time of dementia diagnosis. Furthermore, from the total number of individuals diagnosed with dementia, 122 were diagnosed with Alzheimer's disease.

Participants with missing information were less educated (57\% v. $39.7 \%$ lacking formal qualifications); however, the missing information according to gender, age, occupational class and leisure activities were fairly similar. Participants who developed dementia were significantly older, had lower Cognitive Reserve Index scores, less education, lower-ranked work occupations, engaged less in leisure activities and had less wealth than those who did not develop the condition (see Table 1).

\section{Overall Cognitive Reserve Index and dementia}

The association between Cognitive Reserve Index and dementia incidence across 15 years of follow-up are presented in Table 2. Model 1 of the Cox regression showed that the hazard ratio of the medium level of cognitive reserve was 0.65 (95\% CI $0.53-0.81$, $P<0.001$ ) when compared with the low level after adjustment for gender and marital status, and decreased by $17 \%$ after adjustment for wealth. Similarly, in the minimally adjusted model, the hazard ratio of the high level of cognitive reserve was 0.48 (95\% CI 0.36$0.64, P<0.001)$ when compared with the low level, and decreased by $23 \%$ after adjustment for wealth. The fully adjusted model showed a $27 \%$ decreased risk of dementia for those in the medium level (model 9: adjusted hazard ratio 0.73 , 95\% CI 0.59$0.92, P<0.008$ ), with an overall attenuation of $23 \%$ after adjusting for all risk factors. Individuals in the high level showed a $35 \%$ decreased risk of dementia (model 9: adjusted hazard ratio 0.65, $95 \%$ CI $0.48-0.89, P=0.008$ ), with an overall attenuation of $33 \%$ after adjusting for all risk factors, which suggests an additive effect of covariates. We also explored the interaction between covariates and found an interaction between wealth and CHD $(P=0.04)$, which accounted for some of the difference in the overall PERM. Figure 2 presents the smoothed hazard function of each level of Cognitive Reserve Index.

\section{Cognitive reserve markers and dementia}

As presented in Table 3, in the fully adjusted model, increased years of education predicted a lower risk of dementia for the 50-79 years age group (model 9: adjusted hazard ratio $0.56,95 \%$ CI $0.36-0.88$, $P=0.012$ ), but not for the $\geq 80$ years age group.

For the entire analytical sample, higher occupational class predicted lower risk of dementia in the fully adjusted model, with
Table 1 Baseline characteristics of participants with and without dementia at follow-up

\begin{tabular}{|c|c|c|c|}
\hline & $\begin{array}{l}\text { No dementia } \\
(n=11678)\end{array}$ & $\begin{array}{l}\text { Dementia } \\
(n=602)\end{array}$ & $P$-value \\
\hline \multicolumn{4}{|l|}{ Age, years } \\
\hline $50-59$ & $5029(43)$ & $51(8)$ & \multirow[t]{4}{*}{0.001} \\
\hline $60-69$ & $3540(30)$ & $154(26)$ & \\
\hline 70-79 & $2281(20)$ & $246(41)$ & \\
\hline$\geq 80$ & $828(7)$ & $151(25)$ & \\
\hline \multicolumn{4}{|l|}{ Gender } \\
\hline Men & $5375(46)$ & $251(42)$ & \multirow[t]{2}{*}{0.037} \\
\hline Women & $6303(54)$ & $351(58)$ & \\
\hline Cognitive Reserve Index & $100.8 \pm 14.8$ & $98.8 \pm 14.5$ & 0.001 \\
\hline Low & 3612 (31) & 235 (39) & 0.001 \\
\hline Medium & $3933(34)$ & $192(32)$ & \\
\hline High & $4133(35)$ & $175(29)$ & \\
\hline \multicolumn{4}{|l|}{ Education } \\
\hline 4 years & $4323(37)$ & $321(53)$ & \multirow[t]{4}{*}{0.001} \\
\hline 8 years & $1395(12)$ & $84(14)$ & \\
\hline 12 years & $2848(24)$ & $105(18)$ & \\
\hline 15 years & $3112(27)$ & $92(15)$ & \\
\hline \multicolumn{4}{|l|}{ Occupation } \\
\hline Never worked & $138(1)$ & $17(3)$ & \multirow[t]{6}{*}{0.001} \\
\hline $\begin{array}{l}\text { Low-skilled manual } \\
\text { work }\end{array}$ & $2727(23)$ & $171(28)$ & \\
\hline Skilled manual work & $2027(17)$ & $108(18)$ & \\
\hline Skilled non-manual work & $2786(24)$ & $145(24)$ & \\
\hline Professional occupation & $3283(29)$ & $140(23)$ & \\
\hline Intellectual occupation & $717(6)$ & $21(4)$ & \\
\hline \multicolumn{4}{|l|}{ Leisure activities } \\
\hline Low & $3502(30)$ & $217(36)$ & \multirow[t]{3}{*}{0.001} \\
\hline Medium & 3958 (34) & $210(35)$ & \\
\hline High & $4218(36)$ & $175(29)$ & \\
\hline \multicolumn{4}{|l|}{ Wealth, quintile } \\
\hline 1 (lowest) & $2072(18)$ & $156(26)$ & \multirow[t]{5}{*}{0.001} \\
\hline 2 & 2301 (19) & $130(22)$ & \\
\hline 3 & $2332(20)$ & $124(21)$ & \\
\hline 4 & $2399(21)$ & $98(16)$ & \\
\hline 5 (highest) & $2574(22)$ & $94(15)$ & \\
\hline \multicolumn{4}{|l|}{ Smoking } \\
\hline No & $9538(82)$ & $526(87)$ & \multirow[t]{2}{*}{0.001} \\
\hline Yes & $2140(18)$ & $76(13)$ & \\
\hline \multicolumn{4}{|l|}{ Marital status } \\
\hline Not married & 7890 (68) & $350(58)$ & \multirow[t]{2}{*}{0.001} \\
\hline Married & $3788(32)$ & $252(42)$ & \\
\hline \multicolumn{4}{|l|}{ Depressive symptoms } \\
\hline No & $9810(84)$ & $449(75)$ & \multirow[t]{2}{*}{0.001} \\
\hline Yes & $1868(16)$ & $153(25)$ & \\
\hline \multicolumn{4}{|l|}{$\mathrm{CHD}$} \\
\hline No & $10756(92)$ & $487(81)$ & 0.001 \\
\hline Yes & $922(8)$ & $115(19)$ & \\
\hline Diabetes & & & \\
\hline No & $11048(95)$ & $546(91)$ & 0.001 \\
\hline Yes & $630(5)$ & $56(9)$ & \\
\hline Stroke & & & \\
\hline No & $11322(97)$ & $554(92)$ & 0.001 \\
\hline Yes & $356(3)$ & $48(8)$ & \\
\hline Hypertension & & & \\
\hline No & $8246(71)$ & $346(57)$ & 0.001 \\
\hline Yes & 3432 (29) & $256(43)$ & \\
\hline
\end{tabular}

individuals in the medium level indicating a 30\% decreased risk of dementia compared with those in the lower level (model 9: adjusted hazard ratio $0.70,95 \% \mathrm{CI} 0.57-0.85, P<0.001$ ), and participants in the high level showing a $28 \%$ decreased risk of dementia compared with the lower level (model 9: adjusted hazard ratio $0.72,95 \%$ CI $0.56-0.91, P=0.008$ ). The overall attenuation after adjusting for all risk factors was $17 \%$ for the medium level and $30 \%$ for the higher level. 


\begin{tabular}{|c|c|c|c|c|}
\hline & Cognitive Reserve Index & Hazard ratio $(95 \% \mathrm{Cl})$ & $P$-value & PERM \\
\hline \multirow[t]{3}{*}{ Model 1 (Gender + marital status) } & Low & 1 [Reference] & & \\
\hline & Medium & $0.65(0.53-0.81)$ & $<0.001$ & - \\
\hline & High & $0.48(0.36-0.64)$ & $<0.001$ & - \\
\hline \multirow[t]{3}{*}{ Model 2 (model 1 + wealth) } & Low & 1 [Reference] & & \\
\hline & Medium & $0.71(0.56-0.88)$ & 0.002 & $17 \%$ \\
\hline & High & $0.60(0.44-0.81)$ & 0.001 & $23 \%$ \\
\hline \multirow[t]{3}{*}{ Model 3 (model $1+$ smoking) } & Low & 1 [Reference] & & \\
\hline & Medium & $0.67(0.53-0.83)$ & $<0.001$ & $6 \%$ \\
\hline & High & $0.50(0.37-0.67)$ & $<0.001$ & $4 \%$ \\
\hline \multirow[t]{3}{*}{ Model 4 (model 1+ depressive symptoms) } & Low & 1 [Reference] & & \\
\hline & Medium & $0.68(0.55-0.85)$ & 0.001 & $9 \%$ \\
\hline & High & $0.53(0.40-0.71)$ & $<0.001$ & $10 \%$ \\
\hline \multirow[t]{3}{*}{ Model 5 (model 1 + CHD) } & Low & 1 [Reference] & & \\
\hline & Medium & $0.66(0.53-0.82)$ & $<0.001$ & $3 \%$ \\
\hline & High & $0.49(0.37-0.65)$ & $<0.001$ & $2 \%$ \\
\hline \multirow[t]{3}{*}{ Model 6 (model 1 + diabetes) } & Low & 1 [Reference] & & \\
\hline & Medium & $0.66(0.53-0.82)$ & $<0.001$ & $3 \%$ \\
\hline & High & $0.49(0.37-0.65)$ & $<0.001$ & $2 \%$ \\
\hline \multirow[t]{3}{*}{ Model 7 (model $1+$ stroke) } & Low & 1 [Reference] & & \\
\hline & Medium & $0.66(0.53-0.82)$ & $<0.001$ & $3 \%$ \\
\hline & High & $0.49(0.37-0.65)$ & $<0.001$ & $2 \%$ \\
\hline \multirow[t]{3}{*}{ Model 8 (model 1 + hypertension) } & Low & 1 [Reference] & & \\
\hline & Medium & $0.66(0.53-0.81)$ & $<0.001$ & $3 \%$ \\
\hline & High & $0.49(0.37-0.64)$ & $<0.001$ & $2 \%$ \\
\hline \multirow[t]{3}{*}{ Model 9 (model 1 + all covariates) } & Low & 1 [Reference] & & \\
\hline & Medium & $0.73(0.59-0.92)$ & 0.008 & $23 \%$ \\
\hline & High & $0.65(0.48-0.89)$ & 0.008 & $33 \%$ \\
\hline
\end{tabular}

For the 50-84 years age group, individuals in the higher levels of leisure activity indicated a $26 \%$ decreased dementia risk compared with those in the lower level (model 9: adjusted hazard ratio 0.74, 95\% CI $0.56-0.99, P=0.047$ ), with an attenuation of $40 \%$ after adjusting for all risk factors. For the older age group, leisure activities showed no significant association with dementia incidence.

\section{Sensitivity analyses}

Sensitivity analyses 1 and 2 showed a significant effect of higher cognitive reserve on dementia but not on Alzheimer's disease (Supplementary Tables 4 and 5). Sensitivity analyses 3 and 4 indicated that different thresholds of cognitive reserve did not affect the relationship found between cognitive reserve and dementia incidence (see Supplementary Tables 6 and 7). Sensitivity analysis 5 showed no significant relationship between occupation (estimated from age 40 years) and dementia for the fully adjusted model (see

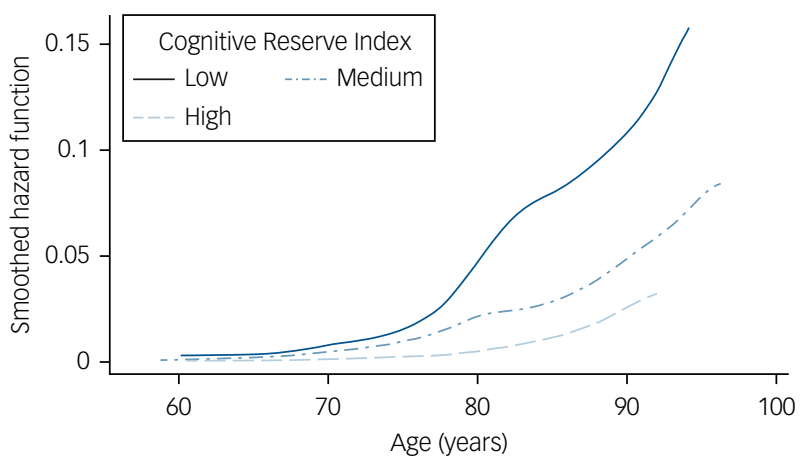

Fig. 2 Adjusted smoothed hazard estimates by levels of Cognitive Reserve Index in the English Longitudinal Study of Ageing.
Supplementary Table 8). Finally, the models controlling independently for alcohol consumption and APOEe4 in sensitivity analysis 6 showed significant associations between cognitive reserve and dementia. However, in the fully adjusted models, the relationship between cognitive reserve and dementia became non-significant (see Supplementary Table 9). The interaction between cognitive reserve and APOEe4 was found to be non-significant $(P=0.155)$.

\section{Discussion}

This study investigated the association between various markers of cognitive reserve by applying a multifaceted index and subsequent dementia incidence in a representative sample of the English population aged $\geq 50$ years. In multivariable analyses, increased levels of Cognitive Reserve Index were negatively and independently associated with dementia incidence when compared with the lowest level. A further sensitivity analysis exploring different thresholds confirmed these results. Our findings suggest a higher risk of dementia for individuals with lower cognitive reserve, evaluated with several markers such as educational attainment, occupational class and engagement in leisure activities. Wealth explained 17$23 \%$ of excess dementia diagnosis in individuals with medium and high levels of Cognitive Reserve Index. Investigation of cognitive reserve markers suggested that low occupational class was associated with higher dementia risk for the entire analytical sample. Education and leisure activities were found to be independently associated with reduced dementia risk only for younger individuals in this cohort.

This study provided a standardised and structuralised index of cognitive reserve, which is replicable and broadly consistent with previous epidemiologic analyses that have found evidence of the protective effects of cognitive reserve markers. ${ }^{17}$ For instance, longitudinal findings from the Cognitive Function and Ageing Study ${ }^{18}$ indicated that a composite score of education, occupation 
Table 3 Hazard ratios from multivariate Cox regressions models indicating the incidence of dementia by each marker of Cognitive Reserve Index

\begin{tabular}{|c|c|c|c|c|c|c|c|}
\hline & & \multicolumn{3}{|c|}{ Age $50-79$ years, $n=9155$} & \multicolumn{3}{|c|}{ Age $\geq 80$ years, $n=3125$} \\
\hline & & Hazard ratio $(95 \% \mathrm{Cl})$ & $P$-value & PERM & Hazard ratio $(95 \% \mathrm{Cl})$ & $P$-value & PERM \\
\hline \multicolumn{8}{|c|}{ Education (years) } \\
\hline \multirow[t]{4}{*}{ Model 1} & 4 & 1 [Reference] & & & 1 [Reference] & & \\
\hline & 8 & $0.93(0.64-1.37)$ & 0.749 & - & $0.89(0.65-1.22)$ & 0.490 & - \\
\hline & 12 & $0.82(0.59-1.14)$ & 0.249 & - & $0.84(0.61-1.17)$ & 0.319 & - \\
\hline & 15 & $0.44(0.29-0.66)$ & $<0.001$ & - & $1.04(0.77-1.42)$ & 0.761 & - \\
\hline \multirow[t]{4}{*}{ Model 2} & 4 & 1 [Reference] & & & 1 [Reference] & & \\
\hline & 8 & $0.98(0.67-1.45)$ & 0.954 & $71 \%$ & $0.94(0.68-1.29)$ & 0.711 & $45 \%$ \\
\hline & 12 & $0.93(0.67-1.31)$ & 0.708 & $61 \%$ & $0.93(0.67-1.30)$ & 0.698 & $56 \%$ \\
\hline & 15 & $0.54(0.34-0.83)$ & 0.006 & $18 \%$ & $1.23(0.90-1.68)$ & 0.187 & $475 \%$ \\
\hline \multirow[t]{4}{*}{ Model 3} & 4 & 1 [Reference] & & & 1 [Reference] & & \\
\hline & 8 & $0.94(0.64-1.38)$ & 0.765 & $14 \%$ & $0.89(0.65-1.23)$ & 0.508 & $0 \%$ \\
\hline & 12 & $0.83(0.60-1.15)$ & 0.269 & $6 \%$ & $0.86(0.62-1.18)$ & 0.364 & $13 \%$ \\
\hline & 15 & $0.44(0.29-0.67)$ & $<0.001$ & $0 \%$ & $1.05(0.78-1.43)$ & 0.717 & $25 \%$ \\
\hline \multirow[t]{4}{*}{ Model 4} & 4 & 1 [Reference] & & & 1 [Reference] & & \\
\hline & 8 & $0.99(0.67-1.46)$ & 0.994 & $86 \%$ & $0.92(0.67-1.26)$ & 0.619 & $27 \%$ \\
\hline & 12 & $0.90(0.64-1.24)$ & 0.530 & $44 \%$ & $0.88(0.64-1.22)$ & 0.475 & $25 \%$ \\
\hline & 15 & $0.49(0.33-0.74)$ & 0.001 & $9 \%$ & $1.09(0.80-1.49)$ & 0.558 & $125 \%$ \\
\hline \multirow[t]{4}{*}{ Model 5} & 4 & 1 [Reference] & & & 1 [Reference] & & \\
\hline & 8 & $0.94(0.64-1.38)$ & 0.768 & $14 \%$ & $0.89(0.65-1.22)$ & 0.508 & $0 \%$ \\
\hline & 12 & $0.85(0.62-1.18)$ & 0.344 & $17 \%$ & $0.85(0.62-1.18)$ & 0.353 & $6 \%$ \\
\hline & 15 & $0.46(0.30-0.69)$ & $<0.001$ & $4 \%$ & $1.05(0.77-1.43)$ & 0.735 & $25 \%$ \\
\hline \multirow[t]{4}{*}{ Model 6} & 4 & 1 [Reference] & & & 1 [Reference] & & \\
\hline & 8 & $0.95(0.65-1.40)$ & 0.828 & $29 \%$ & $0.89(0.65-1.22)$ & 0.491 & $0 \%$ \\
\hline & 12 & $0.84(0.61-1.16)$ & 0.304 & $11 \%$ & $0.85(0.61-1.17)$ & 0.324 & $6 \%$ \\
\hline & 15 & $0.45(0.30-0.67)$ & $<0.001$ & $2 \%$ & $1.05(0.77-1.42)$ & 0.740 & $25 \%$ \\
\hline Model 7 & 4 & 1 [Reference] & & & 1 [Reference] & & \\
\hline & 8 & $0.94(0.64-1.38)$ & 0.770 & $14 \%$ & $0.89(0.65-1.23)$ & 0.509 & $0 \%$ \\
\hline & 12 & $0.83(0.60-1.14)$ & 0.263 & $6 \%$ & $0.85(0.62-1.18)$ & 0.354 & $6 \%$ \\
\hline & 15 & $0.45(0.30-0.67)$ & $<0.001$ & $2 \%$ & $1.05(0.77-1.43)$ & 0.723 & $25 \%$ \\
\hline Model 8 & 4 & 1 [Reference] & & & 1 [Reference] & & \\
\hline & 8 & $0.94(0.64-1.38)$ & 0.782 & $14 \%$ & $0.89(0.65-1.22)$ & 0.484 & $0 \%$ \\
\hline & 12 & $0.84(0.61-1.17)$ & 0.315 & $11 \%$ & $0.84(0.61-1.17)$ & 0.319 & $0 \%$ \\
\hline & 15 & $0.45(0.30-0.68)$ & $<0.001$ & $2 \%$ & $1.04(0.77-1.41)$ & 0.776 & $0 \%$ \\
\hline Model 9 & 4 & 1 [Reference] & & & 1 [Reference] & & \\
\hline & 8 & $1.04(0.70-1.53)$ & 0.833 & $157 \%$ & $0.96(0.70-1.33)$ & 0.843 & $64 \%$ \\
\hline & 12 & $0.99(0.70-1.40)$ & 0.969 & $94 \%$ & $0.98(0.71-1.37)$ & 0.951 & $88 \%$ \\
\hline & 15 & $0.56(0.36-0.88)$ & 0.012 & $21 \%$ & $1.27(0.93-1.74)$ & 0.125 & $575 \%$ \\
\hline Occupation & & Age $\geq 50$ & $n=12280$ & & & & \\
\hline Model 1 & Low & 1 [Reference] & & & & & \\
\hline & Medium & $0.64(0.53-0.78)$ & $<0.001$ & - & & & \\
\hline & High & $0.60(0.48-0.74)$ & $<0.001$ & - & & & \\
\hline Model 2 & Low & 1 [Reference] & & & & & \\
\hline & Medium & $0.68(0.56-0.84)$ & $<0.001$ & $11 \%$ & & & \\
\hline & High & $0.70(0.55-0.88)$ & 0.003 & $25 \%$ & & & \\
\hline Model 3 & Low & 1 [Reference] & & & & & \\
\hline & Medium & $0.65(0.54-0.79)$ & $<0.001$ & $3 \%$ & & & \\
\hline & High & $0.61(0.49-0.76)$ & $<0.001$ & $3 \%$ & & & \\
\hline Model 4 & Low & 1 [Reference] & & & & & \\
\hline & Medium & $0.66(0.55-0.80)$ & $<0.001$ & $6 \%$ & & & \\
\hline & High & $0.63(0.51-0.79)$ & $<0.001$ & $8 \%$ & & & \\
\hline Model 5 & Low & 1 [Reference] & & & & & \\
\hline & Medium & $0.64(0.53-0.78)$ & $<0.001$ & $0 \%$ & & & \\
\hline & High & $0.60(0.48-0.75)$ & $<0.001$ & $0 \%$ & & & \\
\hline Model 6 & Low & 1 [Reference] & & & & & \\
\hline & Medium & $0.65(0.53-0.78)$ & $<0.001$ & $3 \%$ & & & \\
\hline & High & $0.60(0.48-0.75)$ & $<0.001$ & $0 \%$ & & & \\
\hline Model 7 & Low & 1 [Reference] & & & & & \\
\hline & Medium & $0.65(0.53-0.78)$ & $<0.001$ & $3 \%$ & & & \\
\hline & High & $0.60(0.48-0.75)$ & $<0.001$ & $0 \%$ & & & \\
\hline Model 8 & Low & 1 [Reference] & & & & & \\
\hline & Medium & $0.64(0.53-0.78)$ & $<0.001$ & $0 \%$ & & & \\
\hline & High & $0.60(0.48-0.74)$ & $<0.001$ & $0 \%$ & & & \\
\hline Model 9 & Low & 1 [Reference] & & & & & \\
\hline & Medium & $0.70(0.57-0.85)$ & $<0.001$ & $17 \%$ & & & \\
\hline & High & $0.72(0.56-0.91)$ & 0.008 & $30 \%$ & & & \\
\hline Leisure & & Age 50-8 & $n=1069$ & & Age $\geq 8$ & $n=1588$ & \\
\hline Model 1 & Low & 1 [Reference] & & & 1 [Reference] & & \\
\hline & Medium & $0.82(0.64-1.03)$ & 0.099 & - & $0.79(0.56-1.12)$ & 0.190 & - \\
\hline & High & $0.57(0.44-0.73)$ & $<0.001$ & - & $0.70(0.48-1.01)$ & 0.060 & - \\
\hline
\end{tabular}


Table 3 (Continued)

\begin{tabular}{|c|c|c|c|c|c|c|c|}
\hline & & \multicolumn{3}{|c|}{ Age $50-79$ years, $n=9155$} & \multicolumn{3}{|c|}{ Age $\geq 80$ years, $n=3125$} \\
\hline & & Hazard ratio $(95 \% \mathrm{Cl})$ & $P$-value & PERM & Hazard ratio $(95 \% \mathrm{Cl})$ & $P$-value & PERM \\
\hline \multirow[t]{3}{*}{ Model 2} & Low & 1 [Reference] & & & 1 [Reference] & & \\
\hline & Medium & $0.88(0.69-1.13)$ & 0.341 & $33 \%$ & $0.80(0.57-1.13)$ & 0.211 & $5 \%$ \\
\hline & High & $0.69(0.52-0.91)$ & 0.009 & $28 \%$ & $0.73(0.50-1.07)$ & 0.113 & $10 \%$ \\
\hline \multirow[t]{3}{*}{ Model 3} & Low & 1 [Reference] & & & 1 [Reference] & & \\
\hline & Medium & $0.82(0.64-1.04)$ & 0.104 & $0 \%$ & $0.81(0.57-1.14)$ & 0.235 & $10 \%$ \\
\hline & High & $0.57(0.44-0.74)$ & $<0.001$ & $0 \%$ & $0.73(0.50-1.07)$ & 0.111 & $10 \%$ \\
\hline \multirow[t]{3}{*}{ Model 4} & Low & 1 [Reference] & & & 1 [Reference] & & \\
\hline & Medium & $0.87(0.68-1.11)$ & 0.277 & $28 \%$ & $0.81(0.57-1.14)$ & 0.229 & $10 \%$ \\
\hline & High & $0.64(0.49-0.83)$ & 0.001 & $16 \%$ & $0.73(0.50-1.06)$ & 0.102 & $10 \%$ \\
\hline \multirow[t]{3}{*}{ Model 5} & Low & 1 [Reference] & & & 1 [Reference] & & \\
\hline & Medium & $0.81(0.64-1.03)$ & 0.099 & $6 \%$ & $0.79(0.56-1.12)$ & 0.193 & $0 \%$ \\
\hline & High & $0.57(0.44-0.74)$ & $<0.001$ & $0 \%$ & $0.70(0.48-1.02)$ & 0.064 & $0 \%$ \\
\hline \multirow[t]{3}{*}{ Model 6} & Low & 1 [Reference] & & & 1 [Reference] & & \\
\hline & Medium & $0.82(0.65-1.04)$ & 0.111 & $0 \%$ & $0.79(0.56-1.11)$ & 0.189 & $0 \%$ \\
\hline & High & $0.58(0.45-0.74)$ & $<0.001$ & $2 \%$ & $0.70(0.48-1.01)$ & 0.063 & $0 \%$ \\
\hline \multirow[t]{3}{*}{ Model 7} & Low & 1 [Reference] & & & 1 [Reference] & & \\
\hline & Medium & $0.83(0.65-1.05)$ & 0.126 & $6 \%$ & $0.79(0.56-1.12)$ & 0.192 & $0 \%$ \\
\hline & High & $0.58(0.45-0.75)$ & $<0.001$ & $2 \%$ & $0.70(0.48-1.01)$ & 0.061 & $0 \%$ \\
\hline \multirow[t]{3}{*}{ Model 8} & Low & 1 [Reference] & & & 1 [Reference] & & \\
\hline & Medium & $0.82(0.64-1.03)$ & 0.099 & $0 \%$ & $0.79(0.56-1.12)$ & 0.196 & $0 \%$ \\
\hline & High & $0.57(0.44-0.74)$ & $<0.001$ & $0 \%$ & $0.69(0.48-1.01)$ & 0.058 & $3 \%$ \\
\hline \multirow[t]{3}{*}{ Model 9} & Low & 1 [Reference] & & & 1 [Reference] & & \\
\hline & Medium & $0.92(0.72-1.18)$ & 0.542 & $56 \%$ & $0.83(0.59-1.17)$ & 0.303 & $19 \%$ \\
\hline & High & $0.74(0.56-0.99)$ & 0.047 & $40 \%$ & $0.79(0.53-1.17)$ & 0.249 & $30 \%$ \\
\hline
\end{tabular}

and social engagement, similar to the one used in the present study, was protective of dementia, with those with a high score having a $40 \%$ decreased risk of developing dementia compared with those with a low score. ${ }^{19}$ One of the most widespread models regarding the mechanism of action for cognitive reserve suggests that increased levels of cognitive reserve markers moderate between brain pathology and cognitive function. ${ }^{2}$ The level of cognitive reserve can be increased through the engagement in intellectually demanding activities, such as education, non-manual occupations and leisure activities. ${ }^{20}$ It has been suggested that the protective effects of these activities accumulate and act continuously at different stages across life, possibly leading to an increase in the amount of cognitive reserve., ${ }^{5,21}$

Evidence from systematic reviews and a meta-analysis suggest that education contributes to cognitive reserve and that low educational attainment increases the risk of dementia. ${ }^{22}$ However, the present study found an independent relationship between increased education and reduced dementia risk for the 50-79 years age group, but not in the older group. This is in accordance with a recent study carried out in ELSA, showing that socioeconomic disadvantage, and lower wealth rather than low education, was a strong indicator of dementia incidence for individuals born earlier in the 20th century. ${ }^{12}$ These results hint to some potential cohort effects and variation in older individuals educated around the Second World War, when education in England was particularly restrictive (e.g. schools evacuated, teachers in shortage). Further research is needed to clarify the effect of education on cognitive reserve and dementia risk in other intergenerational cohorts with larger population samples to fully disentangle these effects in population subgroups born across different decades.

Furthermore, the current study investigated the independent association between occupation levels as another marker of Cognitive Reserve Index and dementia risk, which was found to be significant. Our results are in concordance with findings from the Whitehall II study, ${ }^{23}$ indicating that higher employment grade, ordered by increasing salary, was protective of cognitive function. ${ }^{24}$ Other studies that have measured occupational complexity for jobs dealing with data, people or things have also indicated a protective effect of work against dementia. ${ }^{25}$ A systematic review of 14 studies investigating the long-term effects of the workplace on dementia concluded that there is evidence for the protective effects of complex occupations dealing with people and data. ${ }^{26}$ However, the contribution of occupation to cognitive reserve remains highly debated with studies yielding conflicting results. ${ }^{2,27}$ A more recent systematic review examining 34 studies found inconclusive results for the association between work activity and dementia risk. ${ }^{22}$ This inconsistency might be caused by the different measures used to assess occupation in different studies.

Leisure activities have been found to be a robust predictor of dementia, with large longitudinal studies showing a lower incidence of dementia ranging between 33 and 52\% for those who engage in various leisure activities. ${ }^{2,28,29}$ We also found that participants who engaged in leisure activities showed a $26 \%$ reduced risk of dementia when compared with those who did not engage, especially in those aged 50-84 years. Other recent longitudinal findings from the Kungsholmen Project in Sweden ${ }^{30}$ indicated that an aggregated measure of late-life leisure activities showed an increased protective effect against dementia compared with early-life and adulthood activities, which included educational and occupational components. ${ }^{31}$ Furthermore, a systematic review exploring 15 longitudinal studies on social networks and leisure activity and their association with dementia risk concluded that social, mental and physical activity could have protective effects against dementia diagnosis by contributing to cognitive reserve. ${ }^{32}$ Therefore, it is plausible that leisure activities involving mental stimulation and those involving physical activity contribute simultaneously to a reduced risk of dementia through neuroprotection by increasing synaptogenesis and enhancing the brain's vasculature. ${ }^{33}$

However, in our study, leisure activities showed no significant association with dementia incidence for the $\geq 85$ years age group; this might be explained by the low number of participants in this age group, reduced engagement in leisure activities or increased neurodegeneration in older age that may surpass cognitive reserve capacity. 
Our sensitivity analysis exploring dementia type indicated that heightened levels of cognitive reserve are negatively and independently associated with the incidence of dementia, but not Alzheimer's disease. We also tested the role of APOEe4 in a sensitivity analysis, and despite a decreased power, this further adjustment did not affect the relationship between cognitive reserve and dementia in the basic adjusted model.

To summarise, our findings support the theory that various cognitive reserve markers, such as occupation and leisure activities, may have the ability to decrease the risk of dementia. To the best of our knowledge, this is the first study to implement a multifaceted index of cognitive reserve to examine its overall effect and the role of each specific marker (e.g. education, occupation and leisure activities) on dementia risk in a representative sample of the English population. Our findings support the theory that cognitive reserve can mitigate the symptoms of neurodegenerative disorders and can potentially buffer dementia onset. ${ }^{3}$

However, some methodological issues should be taken into consideration when interpreting our findings. The first is related to the CRIq implementation in ELSA; this study did not include the number of years spent in work or performing various leisure activities. We partially addressed this aspect by including an estimation of years worked according to baseline age in one of our sensitivity analyses; however, this approach was not appropriate to estimate the element of time for leisure activities. The second limitation is related to the distribution of participants across the levels of Cognitive Reserve Index (see Supplementary Table 2). A third limitation constitutes the use of self-reported dementia diagnosis, which might have resulted in a slight underestimation of the number of participants with dementia in this study. There is also a lack of ethnic variability in the study, as well as a potential attrition bias owing to the longitudinal nature of the study. Lastly, further analyses could take into consideration the time-varying element of both exposures and covariates to better understand how changes in these factors could affect dementia risk.

However, this study has multiple strengths. We used a large, population-based longitudinal study in England to assess multiple markers of cognitive reserve in relation to dementia risk, contributing to the current demand for consistent and replicable methods. ${ }^{5,20,22}$ We provide evidence for the suitability of assessing a multifaceted index of cognitive reserve by using a standardised questionnaire ascertaining multiple markers rather than a singular proxy (e.g. education).

Our findings support the hypothesis that cognitive reserve represents a complex and multifaceted construct, which may have a synergistic influence on dementia risk. Education, occupation and leisure activities were found to be independently related to a reduced risk of dementia, contributing to the accumulation of cognitive reserve across the lifespan. Given that cognitive reserve capacity appears to be determined earlier in life and continues to be enhanced throughout life, this work emphasises the importance of long-life learning and investing in social networks or leisure activities. Our findings also highlight the feasibility of obtaining a standardised, structuralised and replicable index of cognitive reserve in a longitudinal study of ageing. Considering that cognitive reserve is malleable throughout life, public health interventions focusing on increasing brain and mental resilience are recommended to contribute to successful ageing and reduced dementia risk.

Pamela Almeida-Meza, MSc, Department of Behavioural Science and Health, University College London, UK; Andrew Steptoe, DSC, Department of Behavioural Science and Health, University College London, UK; Dorina Cadar (D), PhD, Department of Behavioural Science and Health, University College London, UK

Correspondence: Dorina Cadar. E-mail: d.cadar@ucl.ac.uk

First received 11 Feb 2019, final revision 30 Jan 2020, accepted 10 Feb 2020

\section{Supplementary material}

Supplementary material is available online at https://doi.org/10.1192/bjp.2020.54

\section{Funding}

The work was supported by the National Institute on Aging (grants R01AG7644-01A1 and RO1AG017644); Economic and Social Research Council (grant ES/S013830/1) and Alzheimer Society (grant 477, AS-PhD-18b-022). The English Longitudinal Study of Ageing is funded by the National Institute on Aging (grant R01AG7644) and by a consortium of UK Government departments coordinated by the Economic and Social Research council and the Office for National Statistics.

\section{Acknowledgements}

The authors would like to thank Professor Massimo Nucci, University of Padova, Italy, for providing the formulas for the derivation of the CRIq, and Dr Eddy Davelaar, Birkbeck College, University of London, UK, for implementing the algorithms that were applied to the raw data.

\section{Data availability}

The English Longitudinal Study of Ageing (ELSA) was developed by a team of researchers based at University College London, the Institute for Fiscal Studies and the National Centre for Socia Research. The data are linked to the UK Data Archive and freely available through the UK data Research. The data are linked to the UK Data Archive and freely available
services and can be accessed here: https://discover.ukdataservice.ac.uk.

\section{Author contributions}

P.A.-M. analysed the data, implemented the Cognitive Reserve Index and drafted the manuscript. A.S. designed and conceptualised the study, secured funding and revised the manuscript. D.C. designed and conceptualised the study, derived dementia ascertainment and survival age for each wave, prepared the data, contributed to the implementation of Cognitive Reserve Index and revised the manuscript.

\section{References}

1 Christensen K, Doblhammer G, Rau R, Vaupel JW. Ageing populations: the challenges ahead. Lancet 2009; 374(9696): 1196-208.

2 Stern Y. Cognitive reserve. Neuropsychologia 2009; 47(10): 2015-28.

3 Stern Y. Cognitive reserve in ageing and Alzheimer's disease. Lancet Neurol 2012; 11(11): 1006-12.

4 Barulli D, Stern Y. Efficiency, capacity, compensation, maintenance, plasticity: emerging concepts in cognitive reserve. Trends Cogn Sci 2013; 17(10): 502-9.

5 Opdebeeck C, Martyr A, Clare L. Cognitive reserve and cognitive function in healthy older people: a meta-analysis. Ageing Neuropsychol Cogn 2016; 23(1): 40-60.

6 Nucci M, Mapelli D, Mondini S. Cognitive Reserve Index questionnaire (CRIq): a new instrument for measuring cognitive reserve. Ageing Clin Exp Res 2012; 24 (3): 218-26.

7 Jorm A. A short form of the Informant Questionnaire on Cognitive Decline in the Elderly (IQCODE): development and cross-validation. Psychol Med 1994; 24(1): $145-53$.

8 Quinn TJ, Fearon P, Noel-Storr AH, Young C, McShane R, Stott DJ. Informant Questionnaire on Cognitive Decline in the Elderly (IQCODE) for the diagnosis of dementia within community-dwelling populations. Cochrane Database Syst Rev 2014: (4): CD010079.

9 Office for National Statistics. The National Statistics Socio-Economic Classification (NS-SEC). Office for National Statistics, 2010 (https://www.ons. gov.uk/methodology/classificationsandstandards/otherclassifications/ thenationalstatisticssocioeconomicclassificationnssecrebasedonsoc2010).

10 Fratiglioni L, Viitanen M, von Strauss E, Tontodonati V, Herlitz A, Winblad B. Very old women at highest risk of dementia and Alzheimer's Disease incidence data from the Kungsholmen Project, Stockholm. Neurology 1997; 48 (1): $132-8$

11 Håkansson K, Rovio S, Helkala EL, Vilska AR, Winblad B, Soininen H, et al. Association between mid-life marital status and cognitive function in later life: population-based cohort study. BMJ 2009; 339: b2462.

12 Cadar D, Lassale C, Davies H, Llewellyn DJ, Batty GD, Steptoe A. Individual and area-based socioeconomic factors associated with dementia incidence in England: evidence from a 12-year follow-up in the English Longitudinal Study of Ageing. JAMA Psychiatry 2018; 75(7): 723-32. 
13 Norton S, Matthews FE, Barnes DE, Yaffe K, Brayne C. Potential for primary prevention of Alzheimer's disease: an analysis of population-based data. Lancet Neurol 2014; 13(8): 788-94.

14 Radloff LS. The CES-D scale: A self-report depression scale for research in the general population. Appl Psychol Meas 1977; 1: 385-401.

15 Wolters FJ, Segufa RA, Darweesh SK, Bos D, Ikram MA, Sabayan B, et al. Coronary heart disease, heart failure, and the risk of dementia: a systematic review and meta-analysis. Alzheimer's Dement 2018; 14(11): 1493-504.

16 Elovainio M, Hakulinen C, Pulkki-Råback L, Virtanen M, Josefsson K, Jokela M, et al. Contribution of risk factors to excess mortality in isolated and lonely individuals: an analysis of data from the UK Biobank cohort study. Lancet Public Heal 2017; 2(6): e260-6.

17 Marioni RE, van den Hout A, Valenzuela MJ, Brayne C, Matthews FE. Active cognitive lifestyle associates with cognitive recovery and a reduced risk of cognitive decline. J Alzheimer's Dis 2012; 28(1): 223-30.

18 Brayne C, McCracken C, Matthews FE. Cohort profile: the medical research council Cognitive Function and Ageing Study (CFAS). Int J Epidemiol 2006; 35 1140-5.

19 Valenzuela M, Brayne C, Sachdev P, Wilcock G, Matthews F. Cognitive lifestyle and long-term risk of dementia and survival after diagnosis in a multicenter population-based cohort. Am J Epidemiol 2011; 173(9): 1004-12.

20 Harrison SL, Sajjad A, Bramer WM, Ikram MA, Tiemeier H, Stephan BC Exploring strategies to operationalize cognitive reserve: a systematic review of reviews. J Clin Exp Neuropsychol 2015; 37(3): 253-64.

21 Dekhtyar S, Wang HX, Scott K, Goodman A, Koupil I, Herlitz A. A life-course study of cognitive reserve in dementia-from childhood to old age. Am J Geriatr Psychiatry 2015; 23(9): 885-96.

22 Chapko D, McCormack R, Black C, Staff R, Murray A. Life-course determinants of cognitive reserve (CR) in cognitive aging and dementia-a systematic literature review. Aging Ment Health 2018; 22(8): 915-26.

23 Marmot M, Brunner E. Cohort profile: the Whitehall II study. Int J Epidemio 2005: 34(2): 251-6.

24 Xue B, Cadar D, Fleischmann M, Stansfeld S, Carr E, Kivimäki M, et al. Effect of retirement on cognitive function: the Whitehall II cohort study. Eur J Epidemiol 2018; 33(10): 989-1001.

25 Kröger E, Andel R, Lindsay J, Benounissa Z, Verreault R, Laurin D. Is complexity of work associated with risk of dementia? The Canadian Study of Health and Aging. Am J Epidemiol 2008; 167(7): 820-30.

26 Then F, Luck T, Luppa M, Thinschmidt M, Deckert S, Nieuwenhuijsen K, et al. Systematic review of the effect of the psychosocial working environment on cognition and dementia. Occup Environ Med 2014; 71(5): 358-65.

27 Helmer C, Letenneur L, Rouch I, Richard-Harston S, Barberger-Gateau P, Fabrigoule $\mathrm{C}$, et al. Occupation during life and risk of dementia in French elderly community residents. J Neurol Neurosurg Psychiatry 2001; 71(3): 303-9.

28 Scarmeas N, Levy G, Tang MX, Manly J, Stern Y. Influence of leisure activity on the incidence of Alzheimer's disease. Neurology 2001; 57(12): 2236-42.

29 Wilson RS, De Leon CF, Barnes LL, Schneider JA, Bienias JL, Evans DA, et al. Participation in cognitively stimulating activities and risk of incident Alzheimer disease. JAMA 2002; 287(6): 742-8.

30 Fratiglioni L, Viitanen M, Bäckman L, Sandman PO, Winblad B. Occurrence of dementia in advanced age: the study design of the Kungsholmen Project. Neuroepidemiology 1992; 11(1): 29-36.

31 Wang HX, Karp A, Winblad B, Fratiglioni L. Late-life engagement in social and leisure activities is associated with a decreased risk of dementia: a longitudinal study from the Kungsholmen project. Am J Epidemiol 2002; 155(12): 1081-7.

32 Fratiglioni L, Paillard-Borg S, Winblad B. An active and socially integrated lifestyle in late life might protect against dementia. Lancet Neurol 2004; 3(6): 343-53

33 Churchill JD, Galvez R, colcombe S, Swain RA, Kramer AF, Greenough WT. Exercise, experience and the aging brain. Neurobiol Aging 2002; 23(5): $941-55$ 